\title{
Tubeless video-assisted thoracoscopic surgery in mediastinal tumor resection
}

\author{
Weixue Cui", Danxia Huang", Hengrui Liang, Guilin Peng, Mengyang Liu, Run Li, Xin Xu, Jianxing He \\ Department of Thoracic Surgery and Oncology, the First Affiliated Hospital of Guangzhou Medical University, State Key Laboratory of Respiratory \\ Disease, National Clinical Research Center for Respiratory Disease, Guangzhou Institute of Respiratory Health, Guangzhou, China \\ Contributions: W Cui, D Huang, J He; (II) Administrative support: J He, X Xu; (III) Provision of study materials: W Cui, D Huang, H Liang; (IV) \\ Collection and assembly of data: M Liu, D Huang, R Li; (V) Data analysis and interpretation: W Cui, D Huang, G Peng, X Xu; (VI) Manuscript \\ writing: All authors; (VII) Final approval of manuscript: All authors. \\ \#These authors contributed equally in this work. \\ Correspondence to: Xin Xu; Jianxing He. Department of Thoracic Surgery and Oncology, the First Affiliated Hospital of Guangzhou Medical \\ University, State Key Laboratory of Respiratory Disease, National Clinical Research Center for Respiratory Disease, Guangzhou Institute of \\ Respiratory Health, Guangzhou 510120, China. Email: yichunrenjia@126.com; drjianxing.he@gmail.com.
}

Background It has been reported that tubeless video-assisted thoracoscopic surgery (tubeless-VATS) is feasible and safe for thoracic diseases. Herein, we compared the early outcomes of mediastinal lesion resection between the tubeless and traditional VATS.

Methods: Clinical data of all patients who underwent thoracoscopic mediastinal tumor resection were retrospectively collected. The study involved two groups: tubeless and traditional VATS group. Propensity score matching (PSM) was applied to eliminate the population bias. Intraoperative and postoperative variables were compared among matched cohorts.

Results: In total, 43 patients in the tubeless group and 231 patients in the traditional VATS group were included. After 1:1 PSM, baseline characteristics were comparable. Anesthesia time (177.63 vs. 202.53 min; $\mathrm{P}=0.004)$ was shorter in tubeless group, while operation time (90.95 vs. $101.47 \mathrm{~min} ; \mathrm{P}=0.109)$ was similar. Overall, the total postoperative morbidity rate was similar in the two groups ( $15 \%$ vs. $12.5 \% ; \mathrm{P}=0.556)$. Specially, 4/43 patients in tubeless VATS group need to be re-put chest tubes postoperatively. A significant lower similar level of visual analogue scale score was observed in tubeless VATS group $(1.73 \pm 0.48 v s$. $3.41 \pm 0.87, \mathrm{P}<0.001)$ in postoperative day 1 . Meanwhile, the number of patients using postoperative opioid analgesia was also lower in tubeless VATS group (22.88\% vs. 48.38\%, $\mathrm{P}=0.016)$. Furthermore, hospital duration after surgery ( 2.58 vs. 5.47 days; $\mathrm{P}=0.002$ ) was shorter in tubeless group.

Conclusions: Compared with traditional VATS, tubeless VATS for mediastinal tumor may shorten the anesthesia time, decrease postoperative pain and fasten postoperative recovery in carefully selected patients.

Keywords: Tubeless; video-assisted thoracoscopic surgery (VATS); mediastinal tumor

Submitted Aug 26, 2020. Accepted for publication Feb 04, 2021.

doi: $10.21037 / g s-20-682$

View this article at: http://dx.doi.org/10.21037/gs-20-682 


\section{Introduction}

Mediastinal tumors belong to the heterogeneous group of dysembryoplastic and neoplastic diseases essentially, among which the malignant thymoma, thymic carcinoma and other malignant mediastinal tumors (approximately account for $30 \%$ of all mediastinal tumors) can threaten patients' life (1). For these diseases, the main effective treatment is surgery intervention by far, and a favorable long-term outcome has already been proved (2-4). Video-assisted thoracic surgery (VATS), a minimally invasive technology, has been widely accepted due to various advantages such as smaller incision, shorter hospital stable and less postoperative pain compared with open procedures (3). Reported evidence has also demonstrated the convenience and profit of VATS for mediastinal tumors, either in benign tumor, or in large size/ malignant mediastinal masses (5).

In recent decades, mechanical induced ventilation supported by double-lumen tracheal intubation has been considered as the standard care for mediastinal tumor resection. With the development of VATS techniques and anesthesia technology, tubeless anesthesia with spontaneous ventilation has been widely applied in VATS, so as to avoid the adverse events caused by tracheal intubation, invasive mechanical ventilation and muscle relaxants (6).

As a routine clinical practice, in order to monitor patients' vital sign, intraoperative central venous catheter, urethral catheter and postoperative chest tube were placed. However, trachea intubation may be associated with trachea damage, and central venous catheter is associated with the risk of postoperative infection. Urethra catheterization is associated with urethral injury, and infection even urine retention after extubation, which impairs patient's postoperative rehabilitation (7). Besides, chest tube often causes pain, and increases the risk of postoperative infection that is one of the important factors affecting the length, quality and cost of hospitalization. The absence of these catheters in tubeless-VATS protocol for some selected cases may help patients breath smoothly and reduce pain, which would enhance patients' recovery after surgery strategy (8).

Non-intubated spontaneous ventilation is anesthesia technique, while the tubeless VATS is an enhanced recovery after surgery (ERAS) protocol that includes non-intubated spontaneous ventilation anesthesia, no central venous catheter and urethral catheter during operation and no chest tube after surgery. Our team have demonstrated that non-intubated spontaneous ventilation is safe and may be associated with better short-term outcomes for mediastinum tumor resection (9). However, no comparative study has been conducted to describe the entire tubeless VATS procedure that was applied in mediastinal neoplasms. Hence, we aimed to further analyze the application of tubeless VATS in mediastinal neoplasms surgery.

\section{Methods}

\section{Study design and patient inclusion}

The study was written according to The Strengthening the Reporting of Observational Studies in Epidemiology (STROBE) reporting checklist (10) (available at http://dx.doi. org/10.21037/gs-20-682). The study protocol and methods were approved by the institutional ethics committee of the first affiliated hospital of Guangzhou Medical University (2020-69). The study was conducted in accordance with the Declaration of Helsinki (as revised in 2013).

All patients who underwent mediastinal tumor resection in the first affiliated hospital of Guangzhou Medical University from December 2015 to November 2019 were retrospective collected and included in the primary screening. In terms of patients' selection, we evaluated all patients who could be possibly treated with tubeless VATS. The criteria are as follow: age $<60$, BMI $<25$, no cardiopulmonary dysfunction, ASA status $\leq 2$, and other organs without obvious invasion. Patients who met the criteria were asked to sign an informed consent form to receive tubeless technique surgical. Patients who met the criteria but refused tubeless technique received the conventional intubated surgery. The clinical data of patients were retrospectively identified and collected through electronic medical record. Computed tomography (CT) scans were performed for every patient by two independent radiologists who preoperatively determined the size and location of the lesion. Exclusive criteria: malignant cases with obvious invasion to the surrounding organs, cases that underwent median sternotomy, cases that underwent subxiphoid approach surgery, and cases that received bilateral surgery to both sides. Then, the experimental and the control group were tubeless and routine practice, respectively (Figure 1).

\section{Surgical technique}

The surgical procedure is same between tubeless and traditional group, which was described by Zhao et al. (9). Briefly, the patient with a roll under the shoulder was tilted 


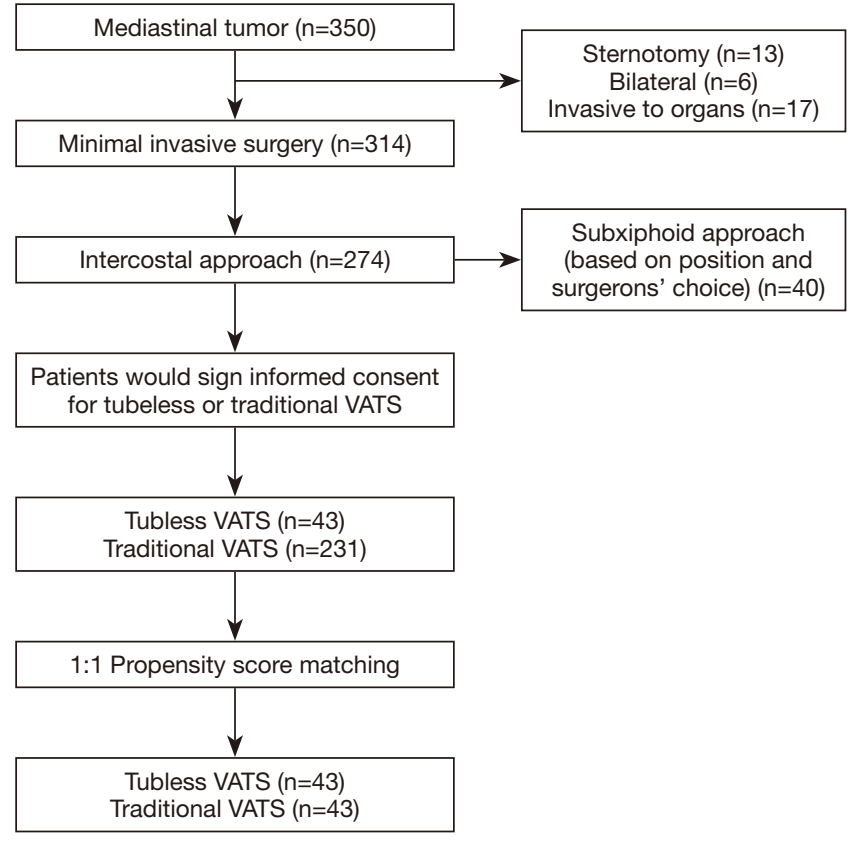

Figure 1 Flow chart of patient inclusion.

$30^{\circ}$ laterally in a semi-supine position. And the ipsilateral arm was held abducted over a padded $\mathrm{L}$ screen to expose the axilla for port placement. The surgical approach was exclusive from the side of the lateral tumor to enable safe dissection under direct vision. Two ports, namely 30 and $15-\mathrm{mm}$, were made. The $30-\mathrm{mm}$ port is for surgical approach and the lower lateral $15-\mathrm{mm}$ port was used for a 30 angled camera. Energy devices (Harmonic Ultracision) were used to reduce bleeding or serous production during surgery. All specimens were safely removed via a specimen bag by enlarging one of the anterior port incisions. Any bleeding or air leakage is treated with a 4/0 PROLENE (Ethicon, Somerville, NJ) suture reinforcement or sealant such as Biopaper (Datsing bio-tech Co., Beijing).

Specially, in traditional VATS group, central venous catheter and urethral catheter and thoracic catheters were placed after anesthesia. A 24-F chest tube was placed at the end of the operation. While, neither of these catheters were placed in tubeless group.

\section{Anesthesia procedure}

Tubeless VATS group: after 3 minutes of anesthesia induction, double-laryngeal mask (FORNIA, Disposable Laryngeal Mask) was placed. After completing the connection of anesthesia machine, we adjusted the breathing parameters as follows: intermittent mandatory ventilation (SIMV) mode VT $6-8 \mathrm{~mL} / \mathrm{kg}$ was synchronized, and RR was 10-12 times/min. In this group, invasive arterial pressure monitor and deep venous catheterization were not used. Subsequently, the patients were induced to spontaneous ventilation to maintain the oxygen flow rate of $4-5 \mathrm{~L} / \mathrm{min}$ and the inhaled oxygen concentration of 70-80\%. Then, mediastinal pleura was opened. Intrathoracic vagus nerve and intercostal nerve were blocked at the beginning of the surgery.

Traditional VATS group: after 3 minutes of anesthesia induction, double lumen tracheal tube was inserted with visual laryngoscope. After completing the connection of anesthesia machine, we adjusted the breathing parameters as follows: tidal volume (VT) $8-10 \mathrm{~mL} / \mathrm{kg}$ and respiration rate (RR) 10-13 times/min in volume-controlled ventilation (VCV) mode. VT 6-8 mL/kg and RR 12-14 times/min in one-lung ventilation afterwards. In this group, invasive arterial pressure monitor and deep venous catheterization (BIOSENSORS INTERNATIONAL TM CV-702-20YT) were used.

If the indicated conditions for conversion, such as hypoxemia or hypercapnia, occur during the surgery, which cannot be resolved after non-invasive management, switching the anesthesia mode and performing tracheal intubation must be carried out by anesthesiologists. The preferred strategy is single-lumen endotracheal intubation + bronchial blockers. If Intra-airway hemorrhage occurs, double lumen endotracheal intubation is the preferred method to achieve pulmonary isolation. It is more difficult than normal practice: Anesthetists insert the single-lumen tube under the guidance of a fiber optic bronchoscope when the patient is in a lateral position. To achieve this, a small pillow should be placed under the patient's head and his or her head and neck should be parallel to the central axis of the body.

\section{Postoperative care}

Patients were resuscitated postoperatively in the resuscitation room and then were returned to the ward. Bedside chest X-ray was performed at the same day after operation and another time before all patients were discharged. Bedside B ultrasound was routinely performed for patients in tubeless group to monitor the pneumothorax or pleural effusion. Patients can resume eating and drinking after bowel sound returned to 5 per min without nausea or vomiting. Two groups of patients were discharged by the following standards: $\mathrm{SaO}_{2}$ on room air $>95 \%$, body $\mathrm{T}<38.0^{\circ} \mathrm{C}$, no respiratory infections, lung re-expansion 
$>70 \%$ after chest tube removal, ultrasound pleural effusion $<8 \mathrm{~cm}$, and no complications requiring in-hospital treatment.

\section{Data collection and statistical analyses}

The re-intubation of chest tube was recorded in tubeless group. The perioperative indexes of the two groups were compared, including operation time, bleeding, re-opening, 30-day morbidity and mortality, lactic acid, duration and volume of thoracic duct, postoperative coagulation function, postoperative arterial blood gas index, postoperative analgesics, length of hospital stay, etc.

The propensity score matching (PSM) generated from the logistic regression was performed to minimize the differences in terms of confounding variables and facilitating matching patients in the two treatment groups (R software version 2.15.1, https://cran.r-project.org/) (11). Variables that could affect the outcomes of treatment were used to generate a propensity score, including gender, age, histology, tumor position and length. Patients were 1:1 matched on the basis of PSM using the nearest-neighbor method on the logit scale. The caliper was set at 0.01 .

Continuous data were presented as mean and standard deviation and were analyzed with 2-sample Student $t$-tests for independent data. Categorical variables were given as a count and the percentage of patients and compared with $\chi^{2}$ or Fisher exact test. All tests were 2-sided, and the a-level of 0.05. SPSS software (SPSS version 25.0; IBM Corp, Armonk, NY) and Graphpad prism 6.0 software were used for all statistical analyses.

\section{Results}

\section{Patients characteristics}

In this study, a total of 350 patients between December 2015 and November 2019 were consecutively included, of which $43(12.29 \%)$ patients received tubeless VATS mediastinal tumor resection (Figure 1). The characters of all patients before PSM are shown in Table 1. After 1:1 PSM, there were 86 (43 vs. 43) patients for analysis, and baseline demographic and clinical variables were fully balanced between the two groups (Table 2).

\section{Operative results and postoperative recovery}

There was no operation-related death. In the tubeless group, no patients required conversion to open surgery during the procedure. In the traditional VATS group, 2/43 (4.65\%) patients were converted to open surgery due to unexpected bleeding. Patients in the tubeless group had similar intraoperative blood loss $(7.79 \pm 6.73$ vs. $8.53 \pm 7.56 \mathrm{~mL}$, $\mathrm{P}=0.361$ ) with traditional practice group. Anesthesia time $(177.63 \pm 84.77$ vs. $202.53 \pm 101.54 \mathrm{~min} ; \mathrm{P}=0.004)$ was shorter in tubeless group, while operation time $(90.95 \pm 63.01 \mathrm{vs}$. $101.47 \pm 56.34$ min; $\mathrm{P}=0.109$ ) was similar.

Overall, the total postoperative morbidity rate was similar in tubeless group and traditional VATS group (15\% vs. $12.5 \% ; \mathrm{P}=0.556)$. Specially, $4 / 43$ (9.3\%) patients in tubeless VATS group need to be re-put a chest tube postoperatively because of pleural fluid $(n=1)$ or atelectasis $(n=3)$. Only 1 patient $(2.3 \%)$ needed to be re-put a chest tube after the extubation of drainage because of pleural fluid in traditional group. Totally, 5/43 (11.6\%) patients in traditional group suffered from sore throat, while only one patient $(2.3 \%)$ in tubeless group has the same symptom. No venous or urinary catheterization-related morbidity was recorded in either group. The average of chest-tube duration was 2.3 days and average drainage volume was $330.13 \mathrm{~mL}$ in traditional VATS group. Furthermore, hospital duration after surgery $(2.58 \pm 1.77$ vs. $5.47 \pm 3.54$ days; $\mathrm{P}=0.002)$ was shorter in tubeless group.

\section{Postoperative arterial blood gases and coagulation function}

Relevant indexes were measured and recorded in the morning of postoperative day 1 . All postoperative coagulation functions were similar in the two groups. However, D-dimer in tubeless VATS group was significantly lower than that in traditional VATS group $(582.25 \pm 647.13$ vs. $1,501.00 \pm 1,298.78 \mu \mathrm{g} / \mathrm{L} ; \mathrm{P}=0.001$ ) (Table 3). The comparison of arterial blood gases between the two groups was not statistically significant (Table 3).

\section{Postoperative analgesia medication}

The comprehensive data of 66 patients (35 tubeless VATS, 31 traditional VATS) were available for the post-operative pain score analysis. A significant lower similar level of visual analogue scale score was observed in tubeless VATS compared with traditional VATS $(1.73 \pm 0.48 v s$. $3.41 \pm 0.87$, $\mathrm{P}<0.001)$ group in postoperative day 1 . Furthermore, the patient number of using postoperative opioid analgesia was also lower in tubeless VATS group (22.88\% vs. $48.38 \%$, $\mathrm{P}=0.016$ ). 
Table 1 Demographic characters of study before 1:1 propensity score matching

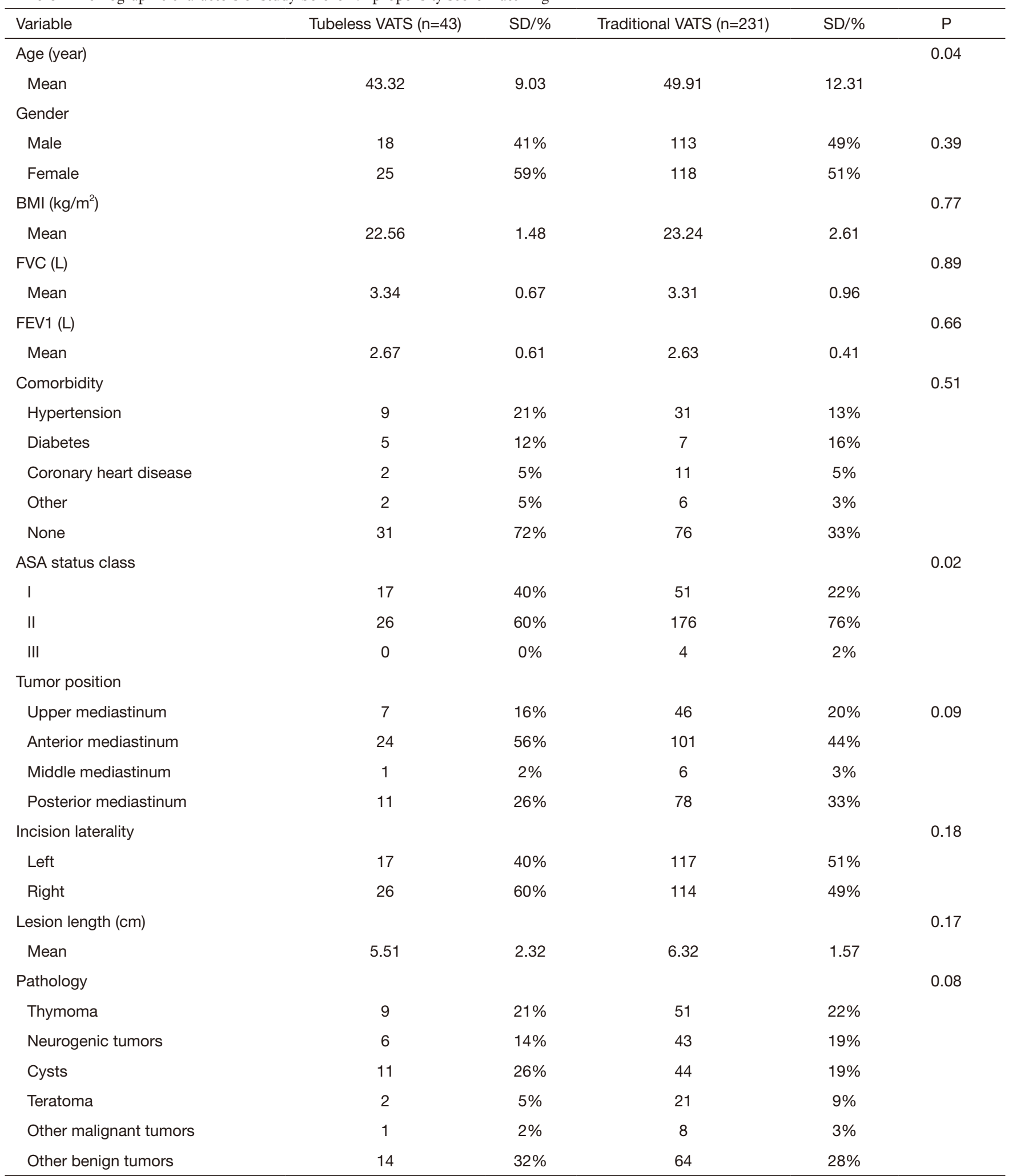

VATS, video-assisted thoracic surgery; BMI, body mass index; FVC, forced vital capacity; FEV1, forced expiratory volume in 1 second; ASA, American Society of Anesthesiologists; SD, standard deviation. 
Table 2 Demographic characters of study after 1:1 propensity score matching

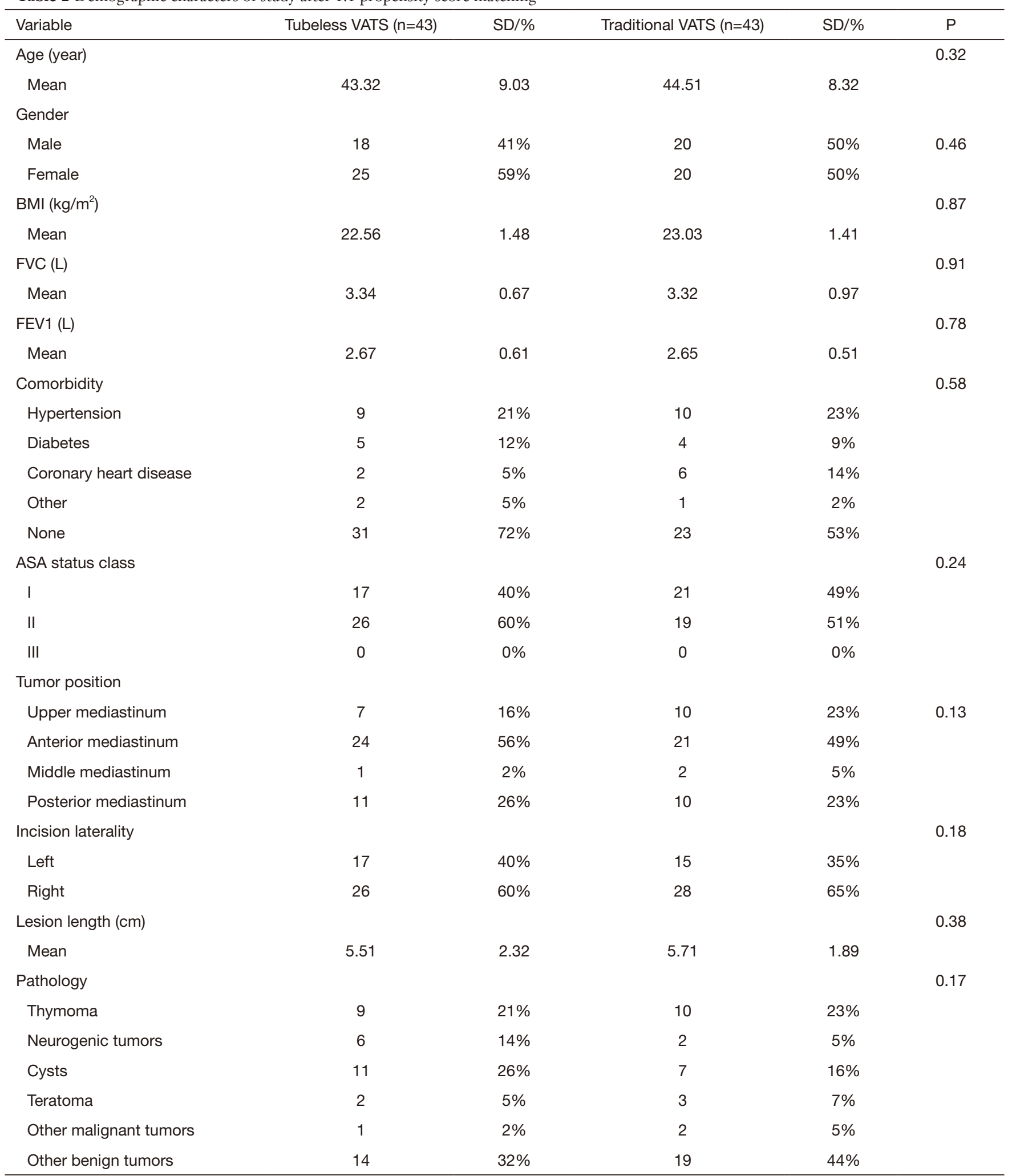

VATS, video-assisted thoracic surgery; BMI, body mass index; FVC, forced vital capacity; FEV1, forced expiratory volume in 1 second; ASA, American Society of Anesthesiologists; SD, standard deviation. 
Table 3 Post-operative index comparison between tubeless and traditional VATS

\begin{tabular}{|c|c|c|c|c|c|}
\hline Variable & Tubeless VATS & SD & Traditional VATS & SD & $\mathrm{P}$ \\
\hline PLT (n/L) & 221.82 & 33.96 & 223.88 & 56.97 & 0.90 \\
\hline PT (s) & 14.22 & 0.42 & 14.54 & 0.86 & 0.43 \\
\hline APTT (s) & 36.08 & 4.71 & 38.24 & 4.67 & 0.38 \\
\hline FIB (g/L) & 3.09 & 1.16 & 3.10 & 0.99 & 0.99 \\
\hline$D$-dimer ( $\mu \mathrm{g} / \mathrm{L})$ & 582.25 & 647.13 & $1,501.00$ & $1,298.78$ & 0.001 \\
\hline \multicolumn{6}{|l|}{ Arterial blood gas } \\
\hline $\mathrm{PH}$ & 7.34 & 0.03 & 7.34 & 0.03 & 0.82 \\
\hline $\mathrm{HCO}_{3}^{-}(\mathrm{mmol} / \mathrm{L})$ & 22.23 & 4.54 & 22.76 & 2.00 & 0.77 \\
\hline $\mathrm{Be}(\mathrm{mmol} / \mathrm{L})$ & -2.97 & 4.36 & -2.42 & 1.94 & 0.75 \\
\hline Lactic acid (mmol/L) & 2.14 & 1.44 & 2.11 & 1.50 & 0.98 \\
\hline
\end{tabular}

VATS, video-assisted thoracic surgery; SD, standard deviation; PLT, platelets; PT, prothrombin time; APTT, activated partial thromboplastin time; TT, thrombin time; FIB, fibrinogen.

\section{Discussion}

Video-assisted thoracic surgery, as a minimally invasive technology, has been shown to be secure in the treatment of mediastinal tumors. Due to the advantages of smaller incision, shorter hospital stays, less postoperative pain and bleeding, this method has been widely accepted in the world (12). With the development of VATS techniques and anesthesia control, tubeless anesthesia with spontaneous ventilation has also gotten better development and application in VATS. The proposition of the tubeless VATS concept aimed to improve patients' experience by minimizing the postoperative pain and discomfort that are associated with interventions. In our study, significant decrease was observed in pain score and hospital duration in tubeless VATS group compared with traditional group. On the premise of ensuring smooth operation, the application of tubeless technique avoids not only complications and pain that are associated with tracheal intubation, chest tubes, and muscle relaxants, but also discomforts at the site of incision and urinary catheters (13).

The spontaneous ventilation anesthesia without tracheal intubation plays an important role in lowering the risk of adverse effects such as intubation-related airway trauma, residual neuromuscular blockade and irritable cough. Besides, with the purpose of maintaining spontaneous ventilation, reducing the dose of analgesic (such as sufentanil) and no muscle relaxant (such as cis-atracurium) were used in tubeless-VATS procedure, which contributes to less anesthesia-related complication and better muscle recovery. Additionally, regional anesthesia therapy decreases invasive movement in the mediastinal cavity, thus allowing a quite stable operative area for surgery (14).

Central venous catheter, urinary catheterization and chest tubes' placement are routinely performed in most thoracic operations. That whether urinary catheterization is indispensable mostly depends on anesthesia and surgical duration. As mentioned above, tubeless-VATS is a special type of non-intubated VATS without using these catheters. Under the absence of central venous catheter, urinary catheterization, ureter discomfort, trauma, the increased risk of infection, and post-removal re-catheterization can be well avoided. Similarly, it is also well recognized that postoperative chest tubes can aggravate postoperative pain, impair ventilation capacity, and affect early ambulation. And ventilator induced diaphragm dysfunction (VIDD), a neuromuscular dyskinesia of diaphragm associated with long-term mechanical ventilation, typically after periods of controlled mechanical ventilation (CMV) 
ventilator mode, can also be avoided with the absence of tracheal intubation (15).

Undoubtedly, difficulty in nursing patients with the placement of chest tubes and urethral catheters is higher than those without tubes. For patients with urethra catheterization, additional jobs might include the cleaning of vulva area, the replacement of catheter periodically, and regular checking to avoid the twist and obstruction of catheter. For patients with insertion of chest tube, maintaining the wound dressings to be clean, preventing the reflux of thoracic drainage fluid, and keeping intrathoracic drainage unobstructed are of high necessity. Tubeless-VATS not only has advantages for patients' intraoperative and postoperative recovery, but also lightens the workload of nursing staff. In order to systematically evaluate the tubeless protocol, we only selected patients that underwent total tubeless VATS technique. A 24-F chest tube was placed at the end of the operation in traditional VATS group. While, neither of these catheters were placed in tubeless group. Whether or not to re-put a chest tube after the operation depends on the patient's status. In order to monitor the postoperative status, routine bedside B-ultrasound was performed every day for tubeless group. However, we found 4 cases of either pleural fluid or atelectasis and needed to re-put a chest tube. This hinted that optimal candidates for tubeless technique should be carefully selected.

The results of this study demonstrated that tubelessVATS significantly shortens the length of stay, which mainly attributes to simplified pre-operation preparation and postoperative nursing. Fewer wounds were proved in the tubeless-VATS group, which is beneficial to postoperative recovery, with less physical function damage to patients. In addition, this method can reduce mental burden, improve patient satisfaction and improve the utilization of existing health resources. In the current thoracic surgery, mechanical induced one-lung ventilation has become the standard care, and intraoperative monitor includes central venous catheters or urethral catheterization has also been deemed as the routine practice in thoracic operation. Surgical treatment lacks individualization and precision. The concept of "tubeless VATS" indicated that simple disease condition should receive simple procedure. This study demonstrated that simple procedure (tubeless VATS) is safe and feasible for simple condition (selected mediastinal tumor resection patients), and the simple procedure is associated with better postoperative short outcomes.

This study also analyzed the characteristics of enrolled patients who underwent tubeless-VATS, compared with those of routine intubated-VATS, and the results demonstrated that patients who received tubeless VATS were younger and had shorter lesion length. Generally, younger patients have better lung function to tolerate anesthesia and do not require strict mechanical ventilation. Furthermore, shorter lesion length means a smaller surgical resection scope and less damage to the surrounding tissues. The choice of tubeless VATS is more conducive to the postoperative recovery of patients. Therefore, for these patients, the absence of a chest tube is also beneficial to their postoperative rehabilitation. Besides, bedside B ultrasound was routinely performed for these patients who underwent tubeless VATS to ensure the intrathoracic safety. For technique requirement, patients who underwent tubeless VATS should be young and have low BMI, smaller tumor and easier condition to avoid poor surgical filed. However, the selection bias would cause the unbalanced outcomes. As the consequence, PSM was used to balance the clinical features pre-operatively, thus leading to a more objective comparison.

During tubeless-VATS surgery, the primary challenge for the anesthetists is the intraoperative conversion of the anesthesia method that is adopted to control over all kinds of complicated situations, such as hypoxemia and hypercapnia (16). As a result of the lack of placement of a chest tube, it is necessary to have careful postoperative monitor, such as bedside B ultrasound. To ensure the security of the processing process, we have developed a comprehensive regulatory access system and a comprehensive security system, including patient selection, preoperative preparation, the operative method, postoperative management, discharge criteria and the followup system. Nevertheless, for mediastinal tumor, tubelessVATS technology is good application prospect.

Several limitations exist in this study. Firstly, this retrospective study is based on historical controls, and the propensity-score matching method could not eliminate all selection bias. Secondly, this study is a single center experience analysis, and the sample size is limited, so multicenter or larger sample size studies are needed. Thirdly, patients' satisfaction cannot be evaluated objectively. Thus, we plan to develop a randomized controlled trial, aiming to compare patients' report outcomes (PROS) between tubeless VATS and traditional group.

\section{Conclusions}

Compared with traditional VATS, tubeless VATS for mediastinal tumor may shorten the anesthesia time, decrease postoperative pain and fasten postoperative 
recovery for carefully selected patients.

\section{Acknowledgments}

Funding: None.

\section{Footnote}

Reporting Checklist: The authors have completed the STROBE reporting checklist. Available at http://dx.doi. org/10.21037/gs-20-682

Data Sharing Statement: Available at http://dx.doi. org/10.21037/gs-20-682

Peer Review File: Available at http://dx.doi.org/10.21037/gs20-682

Conflicts of Interest: All authors have completed the ICMJE uniform disclosure form (available at http://dx.doi. org/10.21037/gs-20-682). The authors have no conflicts of interest to declare.

Ethical Statement: The authors are accountable for all aspects of the work in ensuring that questions related to the accuracy or integrity of any part of the work are appropriately investigated and resolved. The study was conducted in accordance with the Declaration of Helsinki (as revised in 2013). Informed consent has been completed for the publication of this study. The study protocol and methods were approved by the institutional ethics committee of the First Affiliated Hospital of Guangzhou Medical University (2020-69).

Open Access Statement: This is an Open Access article distributed in accordance with the Creative Commons Attribution-NonCommercial-NoDerivs 4.0 International License (CC BY-NC-ND 4.0), which permits the noncommercial replication and distribution of the article with the strict proviso that no changes or edits are made and the original work is properly cited (including links to both the formal publication through the relevant DOI and the license). See: https://creativecommons.org/licenses/by-nc-nd/4.0/.

\section{References}

1. Serra Fortuny M, Gallego M, Berna L, et al. FDG-PET parameters predicting mediastinal malignancy in lung cancer. BMC Pulm Med 2016;16:177.

2. Rabiou S, Lakranbi M, Ghizlane T, et al. Which surgery for mediastinum tumor: Experience of the Department of thoracic surgery of CHU Hassan II of Fes. Rev Pneumol Clin 2017;73:246-52.

3. Peng G, Liu M, Luo Q, et al. Spontaneous ventilation anesthesia combined with uniportal and tubeless thoracoscopic lung biopsy in selected patients with interstitial lung diseases. J Thorac Dis 2017;9:4494-501.

4. Kimura K, Kanzaki R, Kimura T, et al. Long-Term Outcomes After Surgical Resection for Pleural Dissemination of Thymoma. Annals of Surgical Oncology 2019;26:2073-80.

5. Hwang SK, Park SI, Kim YH, et al. Clinical results of surgical resection of mediastinal teratoma: efficacy of video-assisted thoracic surgery. Surg Endosc 2016;30:4065-8.

6. Yang SM, Wang ML, Hung MH, et al. Tubeless Uniportal Thoracoscopic Wedge Resection for Peripheral Lung Nodules. Ann Thorac Surg 2017;103:462-8.

7. Petersen RH, Holbek BL, Hansen HJ, et al. Video-assisted thoracoscopic surgery-taking a step into the future. Eur J Cardiothorac Surg 2017;51:694-5.

8. Cui F, Liu J, Li S, et al. Tubeless video-assisted thoracoscopic surgery (VATS) under non-intubated, intravenous anesthesia with spontaneous ventilation and no placement of chest tube postoperatively. J Thorac Dis 2016;8:2226-32.

9. Zhao Y, Xia Z, Liang W, et al. SARS-CoV-2 Persisted in Lung Tissue Despite Disappearance in Other Clinical Samples. Clinical Microbiology and Infection 2020;26:1424-5.

10. Nagendrababu V, Duncan HF, Fouad AF, et al. Preferred Reporting items for OBservational studies in Endodontics (PROBE): a development protocol. Int Endod J 2020;53:1199-203.

11. Jupiter DC. Propensity Score Matching: Retrospective Randomization? J Foot Ankle Surg 2017;56:417-20.

12. Da M, Peng W, Mo X, et al. Comparison of efficacy between video-assisted thoracoscopic surgery and thoracotomy in children with mediastinal tumors: 6-year experience. Ann Transl Med 2019;7:653.

13. Zhao ZR, Lau RWH, Ng CSH. Anaesthesiology for uniportal VATS: double lumen, single lumen and tubeless. J Vis Surg 2017;3:108.

14. Liang $\mathrm{H}$, Liu J, Wu S, et al. Non-intubated spontaneous ventilation offers better short term outcome for mediastinal tumor surgery. Ann Thorac Surg 2019;108:1045-51. 
15. Marin-Corral J, Dot I, Boguna M, et al. Structural differences in the diaphragm of patients following controlled vs. assisted and spontaneous mechanical ventilation. Intensive Care Med 2019;45:488-500.

Cite this article as: Cui W, Huang D, Liang H, Peng G, Liu M, Li R, Xu X, He J. Tubeless video-assisted thoracoscopic surgery in mediastinal tumor resection. Gland Surg 2021;10(4):1387-1396. doi: 10.21037/gs-20-682
16. He J, Liu J, Zhu C, et al. Expert consensus on tubeless video-assisted thoracoscopic surgery (Guangzhou). J Thorac Dis 2019;11:4101-8. 